\title{
SOBRE LOS FUNDAMENTOS DE LA PROPIEDAD INTELECTUAL*
}

\section{ON THE FOUNDATIONS OF INTELLECTUAL PROPERTY}

\author{
Sulan $\mathrm{WONG}^{* *}$
}

\section{Resumen:}

La discusión sobre los fundamentos que legitiman la propiedad intelectual se suele centrar alrededor de dos grandes planteamientos: iusnaturalistas y utilitaristas. Los primeros sostienen que es posible justificar la propiedad sobre la creación intelectual recurriendo a la teoría liberal de la propiedad formulada por John Locke. Los segundos convergen en torno a la llamada "cláusula del progreso" y el argumento de los incentivos. En este trabajo se analiza si es posible o no justificar la propiedad intelectual sobre la base de dichos planteamientos, concluyendo que, dadas las paradojas identificadas, éstos resultan insuficientes.

\section{Palabras clave:}

Iusnaturalismo; utilitarismo; patrimonio intelectual; privilegio; propiedad.

\section{Abstract:}

The discussion on the foundations that legitimize intellectual property is usually centered around two major approaches: iusnaturalism and utilitarianism. The first one argues that it is possible to justify the property of intellectual creation resorting to the liberal theory of property by John Locke. The second one converges around the so-called "clause of progress» and the argument of the incentives. This paper analyzes whether or not it is possible to

* Artículo recibido el 21 de agosto de 2018 y aceptado para su publicación el 25 de julio de 2019.

** Universidad de La Frontera en Chile, sulan.wong@ufrontera.cl.

Problema. Anuario de Filosofía y Teoría del Derecho, núm. 14, enero-diciembre de 2020, pp. 369-398 Ciudad de México, ISSN 2007-4387, se distribuye bajo una Licencia Creative Commons Reconocimiento-No Comercial-Sin Derivados 4.0 Internacional (CC BY-NC-ND 4.0). 


\section{SULAN WONG}

justify intellectual property under these approaches, concluding that, given the paradoxes identified, they are not sufficient.

\section{Keywords:}

Iusnaturalism; Utilitarianism; Intellectual Commons; Privilege; Monopoly; Property. 
SOBRE LOS FUNDAMENTOS DE LA PROPIEDAD INTELECTUAL

Sumario: I. Introducción. II. Planteamientos iusnaturalistas. III. Planteamientos utilitaristas. IV. Conclusiones. V. Bibliografía.

\section{INTRODUCCIÓN}

La discusión sobre los fundamentos que legitiman la propiedad intelectual se suele centrar alrededor de dos grandes planteamientos: iusnaturalistas y utilitaristas. ${ }^{1}$ Los primeros sostienen que es posible justificar la propiedad intelectual con fundamento en la "teoría de la propiedad" de John Locke, al entenderse que la invención o la creación literaria, artística o científica es el resultado de un proceso intelectual individual, de un esfuerzo sin el que sería imposible conocerla. Es este esfuerzo el que le agrega valor al recurso intelectual - antes no trabajado-, lo transforma en propiedad privada y le libera de toda carga social. Por contra, los segundos convergen en torno a la llamada "cláusula del progreso" y el argumento de los incentivos a la creación intelectual. En este sentido, la necesidad de promover el progreso de la "ciencia y las artes útiles" exige tanto el reconocimiento de la labor creativa del autor o inventor, mediante el establecimiento de derechos de propiedad individual, como la limitación en el tiempo de esos derechos, para recuperar el continuo ciclo de innovación cultural y tecnológica interrumpido por el monopolio conferido.

Con este trabajo no se analiza si Locke le otorgó o no una base moral a la apropiación individual e ilimitada de los recursos entregados

1 De acuerdo con Lemley, hay una tercera forma de justificar la propiedad intelectual: la fe. Ante la falta de evidencia empírica que pueda sostener la hipótesis utilitarista, Merges recurre a su "fe" en la relación causal entre propiedad intelectual y progreso. Lemley acuña esta visión como la "justificación de la propiedad intelectual basada en la fe", equiparándola con una religión cuyo dogma es indiscutible y no necesita demostración. Véase Lemley, Mark A., "Faith-Based Intellectual Property”, UCLA Law Review, 2015, vol. 62, pp. 1337 y 1338; Merges, Robert P., Justifying Intellectual Property, Cambridge, Harvard University Press, 2011, p. 3. No es objeto de este artículo discutir acerca de este fundamento contemporáneo, pues Merges recurre a él únicamente en caso de que el utilitarismo no funcione. 
en comunidad a la humanidad, sino que se intenta determinar si es posible justificar la propiedad sobre la creación intelectual con fundamento en dicha tesis. Por otra parte, se evalúa si los planteamientos utilitaristas, primero, se alejan de la justificación lockeana de la propiedad intelectual, y, segundo, si son suficientes por su cuenta para fundamentar la misma.

\section{PlanteAmiENTOS IUSNATURALISTAS}

En el Segundo tratado sobre el gobierno civil, ${ }^{2}$ John Locke dedica el capítulo $\mathrm{V}$ a fundamentar la propiedad privada, recurriendo a argumentos tanto de orden teológico como utilitarista. ${ }^{3}$ Teológico porque todo tiene su comienzo en Dios. Es él quien ha asignado los frutos y la tierra a la humanidad para que ésta participe en común de ella; por lo tanto, dichos recursos que la tierra produce naturalmente no son privativos de un individuo en particular, sino que per-

2 Locke, John, Segundo tratado sobre el gobierno civil, trad. de Carlos Mellizo, Madrid, Alianza Editorial, 1990/1690. En adelante Segundo tratado.

3 El capítulo V del Segundo tratado ha dado lugar a diversas y encontradas interpretaciones. Tully ha identificado cronológicamente cuatro líneas de interpretación. En el siglo XIX, Locke es interpretado como el padre del socialismo moderno. En la década de 1930 se consolida la interpretación liberal de Locke. Con los trabajos de Strauss y MacPherson se interpreta a Locke como el defensor del "individualismo posesivo" y de la apropiación ilimitada de la riqueza que libera a la propiedad de sus obligaciones sociales. Finalmente, la cuarta línea de interpretación descansaría en la idea de que Locke dejó al Estado la definición de la propiedad. La interpretación particular de Tully sobre Locke se aleja diametralmente a la de MacPherson, al sostener que Locke no dice nada en su teoría acerca de la propiedad privada en el estado de la naturaleza y que no encuentra razón alguna para asumir que el objetivo de Locke era justificar la apropiación ilimitada de los recursos comunales. Véase Tully, James, An Approach to Political Philosophy. Locke in Contexts, New York, Cambridge University Press, 1993, p. 194, y MacPherson, C. B., The political theory of possessive individualism: Hobbes to Locke, New York, Oxford University Press, 1962, p. 310. Por otra parte, Willinsky destaca que Locke, particularmente con respecto a la propiedad intelectual, tenía una mirada utilitarista, pues reivindicó la libertad desde el mercado. Véase Willinsky, John, The Intellectual Properties of Learning: A Prehistory from Saint Jerome to John Locke, Chicago, University of Chicago Press, 2017, p. 305. 
tenecen a la humanidad entera. En origen, según Locke, los recursos de la tierra y sus frutos nos fueron entregados en comunidad, y, por lo tanto, pertenecen a todos. Luego, la ley de la "razón natural" -que también encuentra su origen en Dios- nos impone al mismo tiempo un derecho y una obligación; todos tenemos el derecho de subsistir en el lugar que Dios nos ha colocado. Pero también tenemos la obligación moral de alcanzar los recursos necesarios para garantizar nuestra subsistencia. ${ }^{4}$ En este derecho y obligación de subsistencia - de vivir y no morir - subyace la obligación de trabajar, la piedra angular del discurso de Locke para justificar, por una parte, el derecho natural a la propiedad y, por otra, la ausencia de consentimiento expreso de los comuneros.

Locke, sostiene Drahos, ${ }^{5}$ pudo haber evitado tener que recurrir a enrevesados argumentos para explicar cómo los recursos dados en comunidad se transforman convencionalmente en propiedad privada sin contar, por una parte, con el consentimiento expreso de todos los comuneros y, por la otra, sin desconocer que dichos recursos fueron asignados comunitariamente por Dios. La explicación podría ser la siguiente: partir de una definición de comunidad en términos negativos - la tierra y sus frutos no pertenecen a nadie- más que una en términos positivos - la tierra y sus frutos pertenecen a la humanidad - hubiera eximido a Locke de tener que buscar una justificación a la ausencia de consentimiento expreso. Pero dada la carga teológica de su tesis, recurrir a una definición de comunidad en términos negativos significaba abandonar la concepción cristiana de la creación o establecer la desigualdad en la asignación de los recursos en origen divino. Locke sugiere en sus planteamientos una redefinición del concepto de comunidad en términos positivos, en la que introduce el trabajo como fuente de legitimación de la apropiación individual de los recursos comunales, sin necesidad de contar con el consentimiento expreso de los demás miembros de la comunidad. ${ }^{6}$

4 Locke, op. cit., $\$ 25$.

5 Drahos, Peter, A Philosophy of Intellectual Property, Aldershot, Dartmouth, 1996, pp. 45-47.

6 Tully, op. cit. 
El trabajo, en consecuencia, no sólo es el medio que le permite a las personas cumplir con su obligación moral de alcanzar los recursos necesarios para auto-conservarse, sino que además legitima la apropiación individual de los recursos comunales sin la autorización expresa de todos los comuneros. Es ésta una suerte de "consentimiento tácito" soportado en la premisa del trabajo, dada la dificultad, advertida por Locke, de orden "práctico" y de inviabilidad aparente, de la obtención del consentimiento expreso de parte de todo el género humano.

La lógica de Locke en dicha premisa -imposibilidad de orden práctico y de inviabilidad aparente en la obtención del consentimiento expreso- podría ser la siguiente: supongamos que hay abundantes frutos sobre la tierra y que una persona, antes que otra, se da a la tarea de recogerlos para su sustento. ${ }^{7}$ Pero como los recursos fueron dados en comunidad, esa persona tendría que obtener el consentimiento de todo el género humano. Dicho así, antes de alcanzar el consentimiento la persona muere de hambre. ${ }^{8}$ Ahora, ¿puede alguien realizar reclamo alguno sobre los recursos recogidos porque no se requirió su consentimiento? Locke dice que no. El consentimiento no es necesario, porque lo relevante en la teoría es el esfuerzo que realiza una persona en recoger los frutos para cubrir su obligación de subsistencia. ${ }^{9}$

A partir del trabajo, el ser humano en Locke se perfila como un propietario en sí mismo, uno que agrega valor a los recursos comunales "con el trabajo de su cuerpo y la obra de sus manos", ${ }^{10}$ y los transforma en recursos privados. La legitimidad de la propiedad no está en el consentimiento, sino en el trabajo. El esfuerzo que emplea el hombre en recoger los frutos, cazar la liebre, someter y labrar la tierra, es lo que hace que ya nadie más pueda reclamar derechos de propiedad sobre lo recogido, cazado y labrado. ${ }^{11}$ Por tanto, los recursos ya no son de todo el género humano. Los recursos que en origen

7 Locke, op. cit., §28.

8 Idem.

9 Ibidem, §29.

10 Ibidem, $\$ 44$.

11 Ibidem, $§ 32$. 
son comunales pasan a ser propiedad privada de quien los trabaja, de "aquel que con su esfuerzo los retiró del estado en el que la naturaleza los había dejado". ${ }^{12}$ La tarea de retirar los frutos, el esfuerzo empleado en labrar la tierra para hacerla productiva, es lo que hace que ya nadie más pueda reclamar derechos de propiedad sobre los mismos, ${ }^{13}$ pues no son comunales, y, por tanto, tampoco podría otra persona moralmente pretenderlo. ${ }^{14}$ En suma, el argumento moralista del trabajo le permite a Locke marcar la diferencia entre los recursos pertenecientes a todo el género humano y los que dejan de serlo por modificación, y superar la falta de consentimiento expreso de todos los comuneros. ${ }^{15}$

Los argumentos del filósofo liberal inglés se aproximan al mismo tiempo al utilitarismo, porque aunque los recursos forman parte de la comunidad primigenia, lo comunal no tiene sentido si no representa una utilidad: "Dios ha dado a los hombres el mundo en común; pero como se lo dio para su beneficio y para que sacaran de él lo que más les conviniera para su vida, no podemos suponer que fuese la intención de Dios dejar que el mundo permaneciese comunal y sin cultivar". 16

La utilidad no sólo es concebida en la obra de Locke en términos de subsistencia, sino también en términos de valor agregado a través del trabajo

...que cada uno considere la diferencia que hay entre un acre de tierra en el que se ha plantado tabaco o azúcar, trigo o cebada, y otro acre de esa misma tierra dejado como terreno comunal, sin labranza alguna; veremos, entonces, que la mejora introducida por el trabajo es lo que añade a la tierra cultivada la mayor parte de su valor. ${ }^{17}$

12 Ibidem, $\$ 29$.

13 Ibidem, §32.

14 No obstante, dicha concepción sería probablemente discutida hoy en día por los liberalistas más acérrimos, pues los recursos ociosos, previamente apropiados, jamás serían otorgados en propiedad privada a los más pobres que pudieran utilizarlos, enriquecerlos o explotarlos o darle valor con su trabajo.

15 Ibidem, §34.

16 Locke, op. cit.

17 Ibidem, $\$ 40$. 
Por otra parte, la apropiación individual no disminuye la propiedad comunal, sino que la acrecienta, al hacer la tierra más productiva en beneficio de la humanidad, "pues los frutos que son producidos por un acre de tierra cultivada resultan ser — sin exageración- diez veces más que los producidos por un acre de tierra igualmente fértil que no es aprovechado y continúa siendo comunal". ${ }^{18}$ Incluso, a juicio de Locke, los cálculos de reconocimiento de la utilidad que le aporta el esfuerzo que invierte una persona en el recurso inculto suelen ser modestos, pues prácticamente sin el trabajo el valor de la propiedad comunal es prácticamente "cero". ${ }^{19}$ La utilidad del recurso se debe así al esfuerzo empleado en transformar en útil "algo" que en su estado natural tenía un valor inapreciable.

Los recursos, al ser trabajados, pierden su carácter comunal de origen divino $-\mathrm{y}$ pasan a ser convencionalmente propiedad privada de quien los ha trabajado-,${ }^{20}$ pues los ha dotado de un valor que no poseían en su estado natural. ${ }^{21}$

Por otra parte, la cuantía de lo acumulable es algo que Locke pretende dar por superado en la formulación explícita de dos condiciones: primero, que se deje suficiente ${ }^{22}$ y de igual calidad para los demás, ${ }^{23}$ y segundo, que se tome sólo lo necesario para el sustento sin dejar que se dañe el recurso apropiado. ${ }^{24}$ Dejar suficiente y de igual calidad para los demás responde a ese derecho natural de toda persona de subsistencia; si la tierra y sus frutos fueron asignados a toda la humanidad, todos tienen el derecho a proveerse de dichos recursos para vivir. Por esta misma lógica, se ha de tomar solamente

\section{Ibidem, $\$ 37$.}

19 Sería un cálculo muy modesto decir que los productos de la tierra que le son útiles al hombre nueve décimas partes son el resultado del trabajo. Pues si estimamos justamente las cosas tal y como nos llegan para nuestro uso, y sumamos los diversos gastos, esto es, lo que estrictamente deben a la naturaleza y lo que deben a nuestro trabajo, nos daremos cuenta de que en la mayor parte de ellas el noventa y nueve por ciento debe atribuirse a nuestro esfuerzo. Véase Locke, op. cit., §40.

20 Ibidem, §34.

21 Locke, op. cit., §27.

22 Idem.

23 Ibidem, $\S \S 33-34$.

24 Ibidem, $\S \S 36-37$. 
SOBRE LOS FUNDAMENTOS DE LA PROPIEDAD INTELECTUAL

lo necesario, y tanto lo tomado como el excedente no se deben dejar perder. $^{25}$

\section{Commons intelectual}

A pesar de que Locke no habló sobre la propiedad intelectual en el Segundo tratado, ${ }^{26}$ algunos autores sostienen que es posible extender la tesis del derecho natural a la misma. ${ }^{27}$ Sin embargo, no es una tarea fácil, pues, como bien lo ha afirmado Boyle, va más allá de una simple ecuación basada en la premisa del trabajo. ${ }^{28}$

25 MacPherson, quien ha realizado un minucioso análisis crítico de la teoría de la propiedad de Locke, sostiene que en dicha teoría se formulan explícitamente al menos dos limitaciones a la apropiación individual, pero advierte que existe una tercera, que se considera erróneamente implícita por la lógica de la justificación lockeana. Esta tercera limitación pareciera que viene dada por la cantidad de trabajo empleada por el hombre de Locke para procurarse los bienes necesarios para su subsistencia; extraído este límite de la máxima "el trabajo de su cuerpo y la obra de sus manos", véase MacPherson, op. cit., p. 198. Por otra parte, Bobbio advierte una cuarta limitación a la propiedad derivada de la muerte del trabajador. Esta limitación se explica en el hecho de que siendo solamente el trabajo de la persona lo que da lugar a la propiedad individual, los bienes apropiados no podrían ser heredados y, por tanto, deberían retornar a la comunidad a la cual pertenecían antes de su transformación o apropiación. Véase Bobbio, Norberto, Locke e il diritto naturale, Torino, G. Gappichelli, 1963, p. 235.

${ }^{26}$ Y en efecto, como advierte Willinsky, Locke no sólo apoya la visión newtoniana de la construcción social del conocimiento, sino que no creía que las bases del Segundo tratado fueran adecuadas para justificar la propiedad sobre el conocimiento. Para un excelente recuento histórico acerca del pensamiento de Locke respecto a la propiedad intelectual, véase el capítulo 12 de la obra de Willinsky, op. cit.

27 Hughes, Justin, "The Philosophy of Intellectual Property", Georgetown Law Journal, vol. 77, 1988, pp. 287-366; Moore, Adam, Intellectual Property and Information Control, New Brunswick, NJ Transaction Publishers, 2004, p. 251; Mossof, Adam, "Saving Locke from Marx: The Labor Theory of Value in Intellectual Property Theory”, Social Philosophy and Policy, vol. 29, núm. 2, 2012; Himma, Kenneth, "Toward a Lockean Moral Justification of Legal Protection of Intellectual Property", San Diego Law Review, vol. 49, núm. 1, 2013; Cwik, Bryan, "Labor as the Basis for Intellectual Property Rights”, Ethic Theory Moral Practice, vol. 17, 2014.

28 Boyle, James, The Public Domain. Enclosing the Commons of the Mind, New Haven, Yale University Press, 2008, p. 315. 
La determinación de la existencia en el campo de las ideas de un recurso comunal o commons equivalente al commons material - la tierra y sus frutos - es uno de los más importantes temas a explicar y, tal vez, el más complejo. ${ }^{29}$ Locke sugiere la existencia de un commons positivo e inclusivo, que podríamos entender como ex ante al hombre, es decir, los recursos existentes con independencia del hombre, pertenecientes a todos en comunidad y accesible a todos los comuneros. En el campo de las ideas se presenta la dificultad de explicar la existencia de un commons intelectual ${ }^{30}$ positivo e inclusivo, ex ante al hombre, tal y como lo plantea Locke. Dicha dificultad radica, en primer término, en que al contrario de los recursos materiales que conforman el commons lockeano, los recursos intelectuales son intangibles, inagotables, inapropiables e infinitos, residen en un objeto físico y, además, admiten simultaneidad de uso; cualquier persona en cualquier parte puede, al mismo tiempo, hacer uso del recurso intelectual. ${ }^{31}$ De hecho, la historia da cuenta de una serie de casos en los que dos o más personas han coincidido en sus descubrimientos o invenciones, de manera independiente y separada: Newton (1671) y Leibnitz (1676) con el cálculo; Mendel (1865), De Vries (1900), Correns (1900) y Tschermarck (1900) con las leyes de la herencia; Montgolfier (1783) y Rittenhouse-Hopkins (1783) con el globo aerostático. ${ }^{32}$

En segundo término, para acceder a los recursos intelectuales del commons intelectual ex ante sería necesario contar con conocimiento previo, salvo para un subconjunto de éstos, los más básicos,

29 Drahos, op. cit., pp. 64-83.

30 En este trabajo un commons intelectual hace referencia a los recursos abstractos compartidos por una comunidad, susceptibles de apropiación a través de derechos de propiedad intelectual.

31 Las características enunciadas implican que los límites naturales —suficiencia y despilfarro- a la apropiación ilimitada de los recursos comunales, en el caso de los recursos intelectuales, no siempre se cumplen. Véase Drahos, op. cit., pp. 68 y 69.

32 Para un listado comprensivo de casos en la historia en el que se observa que las mismas ideas han sido producidas de forma independiente por múltiples creadores, véase Ogburn, William y Thomas, Dorothy, "Are Inventions Inevitable? A Note on Social Evolution", The Academy of Political Science, vol. 37, núm. 1, 1922, pp. 83-98. 
que no tendrían necesidad de ello. El conocimiento requiere de conocimiento para ser creado, por lo que es necesario acceder a él de manera libre. Debería conformarse un commons intelectual ex post de donde todos podamos tomar las ideas necesarias para extraer recursos intelectuales del commons intelectual ex ante. Pero ¿cómo podría configurarse un commons intelectual ex post, en los términos necesarios, es decir, positivo e inclusivo, si los recursos intelectuales requeridos ya han sido apropiados al ser extraídos del commons intelectual ex ante? Nos encontramos frente a una paradoja que cuestionaría la idea de propiedad intelectual basada en Locke, pues el commons intelectual ex ante perdería su cualidad inclusiva. Consideramos, como lo hace Drahos, ${ }^{33}$ que el reto de cara a la tesis lockeana estaría en explicar cómo esos recursos intelectuales pasarían a formar parte del commons intelectual ex post, pues la conformación de este commons exige un proceso inverso, de privado a comunal, que dependería de la voluntad de los comuneros.

\section{El trabajo y el valor agregado}

Locke ha planteado que los recursos comunales devienen en propiedad privada en el momento en que alguien a través de su trabajo les agrega un valor que antes no tenían. Extender este planteamiento a la propiedad intelectual encierra al menos dos problemas; el primero relacionado con la determinación del valor agregado, y, el segundo, vinculado al "comportamiento" de los recursos intelectuales.

Hettinger ${ }^{34}$ ha señalado que la determinación del valor agregado, aunque se manifiesta como un problema de orden práctico, parece obedecer a la errónea tendencia dirigida a simplificar el valor agregado "trabajo" en el valor "producto intelectual" del trabajo. Dado que los recursos o "productos" intelectuales son el resultado de un proceso colectivo en el que han intervenido muchas personas en el

33 Drahos, op. cit., p. 49.

34 Hettinger, Edwin C., "Justifying Intellectual Property", Philosophy and Public Affairs, vol. 18, núm. 1, 1989, pp. 31-52. 
tiempo, ${ }^{35}$ con dicha simplificación lo que se hace es atribuir al último eslabón en la cadena de creación el cien por ciento del valor del trabajo intelectual. ${ }^{36}$ En segundo término, los recursos intelectuales, a diferencia de los recursos materiales, se "comportan" de manera diferente, debido a que no se agotan con el uso. Además, obedecen al llamado "efecto red" o Ley de Metcalfe, por lo que los recursos del commons intelectual ex post adquieren más valor a medida que más personas lo utilizan. Este "extraño lockeanismo" ${ }^{37}$ que es observado, tanto en la red telefónica como en la ciencia, exige la apertura del acceso del commons intelectual a todo aquel que desee usarlo.

La comunidad software libre puede ser vista como el caso paradigmático de la conformación de commons intelectual ex post, donde tanto los creadores como los usuarios colaboran en el mejoramiento y ampliación de la base de conocimiento disponible. Desde la lógica del software libre, el trabajo se entiende de una forma distinta a la tesis lockeana; el trabajo es una actividad intrínsecamente interesante, inspiradora y gratificante. ${ }^{38}$ Mediante múltiples proyectos, que van desde el sistema operativo GNU/Linux a cualquier tipo de servicio o aplicación informática, el movimiento del software libre, por ejemplo, ha logrado conseguir la mayor parte del mercado de los servidores de páginas web que hay en Internet. ${ }^{39}$ El éxito de esta comunidad tiene mucho que ver con los excelentes canales de retroalimentación entre usuarios y desarrolladores, que permiten mo-

35 La concepción newtoniana de la creación como proceso colectivo — si puedo ver más allá es porque estoy parado sobre hombros de gigantes - siempre ha encontrado sus detractores. Véase Spooner, Lysander, The Law of Intellectual Property; or an essay on the right of authors and inventors to a perpetual property in their ideas, Boston, Bela Marsh, 1885, vol. I, Objection sixth.

36 En la práctica, el problema de la determinación de la proporción económica del aporte individual tampoco es sencillo de resolver, y para algunos se manifiesta insostenible. Véase Mossof, op. cit.

37 Rose, Carol, Property \& Persuasion: Essays on the History, Theory, and Rhetoric of Ownership, Boulder, Westview Press, 1994, p. 317.

38 Himanen, Pekka, The Hacker Ethic and the Spirit of the Information Age, New York, Random House, 2001, p. 232.

39 Datos recientes pueden verse en Netcraft, February 2018 Web Server Survey, disponible en: https://news.netcraft.com/archives/2018/02/13/february-2018-webserver-survey.html 
SOBRE LOS FUNDAMENTOS DE LA PROPIEDAD INTELECTUAL

dificaciones, actualizaciones e innovaciones de los recursos con frecuencia. El software libre con su Licencia Pública General, conocida por sus siglas en inglés como GPL, ha sabido ir de la esfera privada a la comunal sin apartarse, paradójicamente, de los pilares fundamentales de la propiedad intelectual. ${ }^{40}$

\section{Suficiente y de igual calidad}

La apropiación de los recursos materiales del commons ex ante en la teoría de Locke está condicionada, primero, a que se deje "suficiente y de igual calidad" para los demás, y, segundo, a que se tome sólo lo necesario para el sustento, sin dejar que se dañe el recurso apropiado.

Entender que, aunque finitos, hay recursos materiales "suficientes y de igual calidad para todos" es un supuesto que Locke estableció para justificar la apropiación de recursos por aquel que los trabaje, pues nunca se podría perjudicar con esta acción al resto de la humanidad. ${ }^{41}$ Pero los recursos intelectuales son infinitos, en el sentido de que siempre habrá ideas por descubrir. Hughes ${ }^{42}$ y Moore $^{43}$

40 Usando el poder del copyright, el "software libre" no sólo asegura que permanece abierto y susceptible de modificación, sino también que otro software que incorpore y use "software libre" - y que técnicamente se convierta en "obra derivada" - debe también, a su vez, ser libre. Si uno usa y adapta un programa de software libre y distribuye públicamente esa versión adaptada, la versión distribuida debe ser tan libre como la versión de la que procede. Debe hacerse así, de lo contrario, para Lessig, se estaría infringiendo el copyright. Véase Lessig, Lawrence, "Introducción", en Stallman, Richard, Software libre para una sociedad libre, Madrid, Traficantes de Sueños, 2004, p. 12.

41 "Mas, sea como fuere, pues no voy a insistir en esto, lo que sí me atrevo a afirmar sin duda alguna es lo siguiente: que esa misma regla de la propiedad, a saber, que cada hombre sólo debe posesionarse de aquello que le es posible usar, puede seguir aplicándose en el mundo sin perjuicio de nadie; pues hay en el mundo tierra suficiente para abastecer el doble de sus habitantes". Cfr. Locke, op. cit., §9.

42 Hughes, op. cit.

43 Moore ha argumentado sobre la posibilidad de justificar la propiedad intelectual a través de la teoría de la propiedad de Locke, dado que la frontera de la propiedad intelectual es prácticamente infinita. En su opinión, la metáfora del bebedor de agua del pasaje 33 del Segundo tratado es muy parecida a la metáfora del autor o 
sugieren que esta característica justificaría incluso más la apropiación de los recursos intelectuales en los términos de Locke, pues al ser éstos infinitos siempre habrá "suficiente y de igual calidad" para los demás. No obstante, se puede discrepar de esta afirmación, pues las condiciones fijadas por Locke no son aplicables estrictamente en el caso de los recursos intelectuales, debido a la naturaleza de los mismos.

Primero, se entiende que en el commons material, aunque en términos finitos, hay "suficiente y de la misma calidad" de cada recurso para todos, es decir, hay múltiples elementos del mismo recurso, pero no existe una diferencia determinante entre ellos. Daría lo mismo tomar cualquier manzana de un huerto, pues ellas son, a todos los efectos, iguales; cuando se extrae una manzana, no deja de haber manzanas. Por contra, aunque los recursos en el commons intelectual ex ante son infinitos, podemos decir que cada recurso intelectual tiene una única forma de materializarse, es un elemento único en un conjunto infinito de elementos únicos. Pueden buscarse otros recursos intelectuales que sean similares al primero y que hasta cumplan la misma función, pero nunca serán el mismo. Por lo tanto, será imposible ${ }^{44}$ para alguien más volver a extraer este recurso desde el commons intelectual ex ante, pues ya ha sido apropiado por quien lo ha hecho primero. Volviendo al caso de las manzanas, es como si al extraer una manzana del commons material ya no se pudieran volver a extraer manzanas nunca más.

Segundo, existen situaciones en las cuales algunos recursos no pueden ser extraídos del commons intelectual ex ante, pues esa ac-

inventor, porque sacar "algo" del commons intelectual es como tomar agua del río. El recurso, en ambos casos, se presenta infinito. Su argumento se apoya en la prueba lógica de la "línea base": si al apropiarnos de un recurso no dejamos en peor condición a los demás en términos de bienestar, entonces la apropiación y la exclusión son permitidas. Véase Moore, op. cit.

44 Es cierto, como ya se comentó anteriormente, que dos personas pueden llegar a un mismo resultado científico o tecnológico de manera independiente. No obstante, esto se hace por desconocimiento del hecho, ya que si la propiedad fuese instantánea sería imposible hacerlo. Lo que, es más, en casos contemporáneos siempre habrá disputa por la prioridad, con lo que quien la demuestre habrá excluido a los demás. 
ción depende de otro recurso previamente extraído por un tercero. ${ }^{45}$ Esto ocurre cuando se toma un recurso del commons intelectual ex ante y no se coloca en el commons intelectual ex post; los recursos dependientes serán indirectamente apropiados por quien detente la propiedad del recurso necesario. Aún más, dado que el commons intelectual ex ante es infinito, la cantidad de recursos intelectuales en éste que dependen de un recurso intelectual extraído puede también ser infinita. Por tanto, este propietario no habrá dejado en el commons "suficiente y de igual calidad" para todos, pues nadie que no sea él podrá realizar trabajo creativo o inventivo derivado del recurso apropiado.

Finalmente, recordemos que la teoría de Locke exige que lo que se tome del commons no se despilfarre. Sin embargo, el propietario de un recurso intelectual no tiene por qué darle utilidad al mismo. Puede haberlo extraído con el único fin de evitar que alguien más lo hiciera o para tener control monopólico del mismo. Es cierto que, en la teoría de la utilidad económica, el propietario de este caso podría extraer valor por el mero hecho de tener su propiedad. Pero es de difícil justificación que esta utilidad sea tan grande como si el recurso intelectual extraído se pusiera a producir o se colocara en el commons intelectual ex post. Además, las ideas se "deterioran" o se "desperdician", 46 en el sentido de que una vez apropiadas, el tiempo durante el que la aplicación de la misma puede ser útil es limitado. Por tanto, dejar de usar un recurso extraído del commons intelectual ex ante violaría el "proviso" lockeano.

\section{Planteamientos UtilitaRistas}

\section{Condorcet y la noción social de las ideas}

La historia de la propiedad intelectual no se inicia en términos de derechos en el sentido moderno, sino en términos de concesiones

45 La investigación desarrollada a partir del descubrimiento del ADN podría ser un buen punto de discusión sobre el tema.

${ }^{46}$ Hettinger, op. cit., pp. 31-52, y Drahos, op. cit., pp. 46-68. 
o privilegios reales otorgados con claros objetivos económicos, sociales y políticos. ${ }^{47}$ Con la invención de la imprenta, cuando Venecia contaba con un gran centro tipográfico, se comienza a plantear la distinción entre la obra intelectual y el soporte material en el que se ha exteriorizado la obra, surgiendo de esta manera el problema de la reproducción de las obras intelectuales. Sin embargo, la actividad de difusión de dichas obras quedaba sujeta a las actividades económicas, subordinadas éstas a los permisos conferidos por las autoridades o la pertenencia a una corporación. ${ }^{48}$ Esta práctica italiana de garantía de privilegio se extendió hacia Francia e Inglaterra. Así, se observa que en la época de Isabel I en Inglaterra se comisiona a la Stationers' Company como la cofradía autorizada para la impresión de libros licenciados en el reino. ${ }^{49}$

Los derechos de beneficios económicos se derivaban no de la propiedad de las ideas, sino de un "privilegio" extendido solamente por la "gracia" real, que funcionaba a la vez como una aprobación oficial de la obra y un permiso exclusivo de reproducción. ${ }^{50}$ El autor, como no podía publicar sus propios libros, lo que obtenía era un beneficio único con la venta de su manuscrito a un editor licenciado, y el verdadero beneficio llegaba con la obtención de un patronazgo real o aristocrático. Por otro lado, la patente se concedía en Europa con la finalidad de impulsar el desarrollo de la industria textil. ${ }^{51}$ También se usó como instrumento real para evadir el control parlamentario de los impuestos y para oscurecer el patrimonio económico de la Corona. El tiempo para la explotación de la patente no seguía una regla

47 Para un recuento sobre como la propiedad intelectual funcionaba como una herramienta de censura y los argumentos utilizados para mantenerla giraban en torno a esta cualidad, recomendamos la obra de Willinsky, op. cit., p. 300.

48 Ascarelli, Tulio, Teoría de la concurrencia y de los bienes inmateriales, 3a., ed., trad. de Evelio Verdera (caps. I a VI) y Luis Suárez-Llanos (caps. VII y ss.), Barcelona, Bosch, 1970.

49 Hesse, Carol, "Enlightenment Epistemology and the Laws of Authorship in Revolutionary France”, 1777-1793, Representations, núm. 30, 1990.

50 Ibidem, p. 111.

51 Wyndham, Hulme, "History of the Patent System under the Prerogative and at Common Law", Law Quarterly Review, vol. 12, 1896; Bracha, Oren, "The Commodification of patents 1600-1836: How patents became rights and why we should care", Loyola of Los Angeles Law Review, vol. 38, 2004-2005. 
SOBRE LOS FUNDAMENTOS DE LA PROPIEDAD INTELECTUAL

fija; se otorgaron patentes por diez, veinte y hasta treinta años. La naturaleza y condiciones para la concesión también era variada: en algunas se protegía el derecho de los usuarios de las máquinas preexistentes, otras protegían las mejoras subsecuentes de las invenciones, algunas requerían el empleo de aprendices y, en el caso de las patentes concedidas a extranjeros, se exigía el empleo de aprendices "nativos". Esta última exigencia como una forma de garantizar la transferencia del conocimiento técnico. ${ }^{52}$

En Francia, el interés por marcar una línea divisoria entre Ancien Régime y la fundación de la República suponía la abrogación de todos los privilegios reales. En este contexto, y en la discusión sobre la cuestión de la propriété littéraire, surge entre Diderot y Condorcet un interesante debate que, como bien lo ha puesto de manifiesto Hesse ${ }^{53}$ reveló una tensión inherente a la epistemología de la Ilustración acerca del origen de las ideas.

Diderot defendió la propiedad sobre las ideas como un derecho natural, ${ }^{54}$ por lo que el autor nada debe a la sociedad; lo que ha obtenido lo ha logrado a través de su educación y de su tiempo, de su investigación y de sus sentimientos, de su dedicación y de su trabajo. El creador e inventor, al igual que el recolector lockeano, realiza un esfuerzo para dotar de valor a las ideas que antes del proceso

52 Entre los autores que han tratado abundantemente el tema desde una perspectiva historiográfica, véase Federico, P. J., "Origin and Early History of Patents", Journal of the Patent Office Society, vol. 11, 1929; Ramsey, George, "The Historical Background of Patents", Journal of the Patent Office Society, vol. 18, 1936; Prager, Frank, "Brunelleschi's Patent", Journal of the Patent Office Society, vol. 28, 1946; Getz, L., "History of the Patentee's Obligations in Great Britain: Part I", Journal of the Patent Office Society, vol. 46, 1964; Pollack, Malla, "The Multiple Unconstitutionality of Business Method Patents: Common Sense, Congressional Choice, and Constitutional History", Rutgers Computer \& Tecnology Law Journal, vol. 28, 2002; Mgbeoj Ikechi, "The Juridical Origins of the International Patent System: Towards a Historiography of the Role of Patents in Industrialization", Journal of the History of international Law, vol. 5, 2003; Bracha, op. cit.

53 Hesse, op. cit.

${ }^{54} \mathrm{Al}$ igual que Diderot, los editores de libros se apoyaron en Locke para insertar a la propiedad intelectual en el discurso de los derechos naturales. Por lo tanto, los privilegios no eran más que el reconocimiento de un derecho anterior fundado en el trabajo del autor, cedido a los editores a través de un contrato de edición. Véase Hesse, op. cit., p. 112. 
creativo no poseían. Incluso, se trata de una propiedad que, al ser difícilmente inseparable del individuo, pues la entiende como una porción de él mismo, la coloca en un plano superior de exigibilidad con respecto a cualquier otra forma de propiedad, pues no perece y, además, inmortaliza al individuo. ${ }^{55}$ La concepción de Diderot sobre las ideas en defensa de la propiedad intelectual como inherentemente subjetivas e individuales ${ }^{56}$ colabora con la inscripción de la creación intelectual en el relato jurídico-político moderno liberal de los derechos y, al mismo tiempo, anula la sociabilidad de los seres humanos. ${ }^{57}$

Condorcet presenta una visión completamente antagónica a la expuesta por Diderot y los partidarios del iusnaturalismo racionalista, introduciendo la noción social de las ideas.

En primer término, no asimila los recursos materiales a los recursos intelectuales, sino que los diferencia en origen. La propiedad sobre los primeros la define como una "propiedad derivada de un orden natural y defendida por la fuerza social”, mientras que la propiedad sobre los segundos es para él "una propiedad fundada por la sociedad misma". ${ }^{8}$ Por tanto, las ideas no son concebidas como el resultado de un proceso individual, sino como el fruto de un pro-

55 "[Q]uel est le bien qui puisse appartenir à un homme, si un ouvrage d'esprit, le fruit unique de son éducation, de ses études, de son temps, de ses veilles, de ses recherches, de ses observations, si les plus belles heures, les plus beaux moments de sa vie, si ses propres pensées, les sentiments de son cœur, la portion de lui-même la plus précieuse, celle qui ne périt point, celle qui l'immortalise, ne lui appartient pas?". Cfr. Diderot, Dennis, Lettre sur le comerce de la librairie: Lettre historique et politique adressée à un magistrat sur le commerce de la librairie, son état ancien et actuel, ses règlemnets, ses privilèges, les permissions tacites, les censeurs, les colporteurs, le passage des ponts et autres objets relatifs à la police littéraire, ed. por Paillard, C. Chicoutimi, Université du Québec à Chicoutimi, 2002/1763, disponible en: http://classiques.uqac.ca/classiques/Diderot_denis/lettre_commer ce_livre/lettre_com_livre.html

56 Hesse, op. cit., p.117.

57 Capella, Juan Ramón, Fruta prohibida: una aproximación histórico-teorética al estudio del derecho y del Estado, 5a., ed., Madrid, Trotta, 1997, p. 361.

58 Condorcet, Marquis de J. A. N.-de-C., "Fragments sur la liberté de la presse", en Oeuvres de Condorcet, París, Didot, 1776, t. II, p. 309, disponible en: http://archivio marini.sp.unipi.it/78/. 
SOBRE LOS FUNDAMENTOS DE LA PROPIEDAD INTELECTUAL

ceso colectivo de aprendizaje. Condorcet sustrae a la obra o creación intelectual de premisas individualistas y, al contrario de Diderot, las considera inherentemente objetivas y sociales. La propiedad intelectual no es un verdadero derecho natural, sino un privilegio tolerado por la sociedad para garantizar que el autor, aunque no hace libros por dinero, ${ }^{59}$ pueda vivir de su obra, ${ }^{60}$ y la sociedad pueda beneficiarse de su aporte, promoviendo el progreso y el bien común.

En segundo término, advierte que, en términos generales, los privilegios tienen el inconveniente de concentrar la actividad en pocas áreas y manos, creando escasez, elevando los precios y reduciendo la calidad de los productos, por lo que para él era importante analizar si éstos eran realmente necesarios y útiles. El progreso alcanzado al margen de los privilegios le permite a Condorcet cuestionar la posible relación entre ambos, ${ }^{61}$ refutando su necesidad como incentivo. Tampoco los encuentra útiles, ya que el autor de una obra no podría impedir que otros expusieran las "verdades útiles" que él ha revelado y, además, nada tendría que reprochar a quien pretenda hacerlo en tanto reconozca su autoría. ${ }^{62}$

Sin embargo, las ideas de Condorcet y Diderot parecen conciliarse en el texto de la propuesta legislativa que Sieyès presenta ante la

59 Ibidem, p. 310.

${ }^{60}$ Aunque el filósofo francés no explica en Fragments sur la liberté de la presse cómo los autores podrían proveerse de los medios de subsistencia por su trabajo intelectual, se puede inferir de su relato que de alguna forma hacía referencia a la figura del mecenazgo como fórmula alternativa en la habilitación de esta posibilidad.

61 “Quand bien même il n'existerait pas de priviléges en librairie, Bacon n'en eût pas moins enseigné la route de la verité dans les sciences; Képler, Galilée, Huyghens, Descartes, nén eussent pas moins faite leurs découvertes; Newton n'en pas moins trouvé le système du monde; M. D’Alembert n'en eût pas moins résolu le problème de la précession des équinoxes. Les decouvertes de la circulation du sang, de l'írritabilité; les recherches heureuses des Satahl, des Bergman, des Scheele, des Priestley, ne son pas le fruit des priviléges en librairie. Dans d'autres géneres, les ouvrages qui on le plus contribié au progrès de lumières, l'Encyclopédie, les oeuvres de Montesquieu, de Voltaire, de Rousseau, nònt pas joui des avantages du privilége". Cfr. Condorcet, op. cit., p. 309.

${ }^{62}$ Condorcet se estaría aquí refiriendo al hoy configurado como "derecho de paternidad de la obra", reconocido en algunas normas de propiedad intelectual, y que vincula al autor con su obra. 
Asamblea el 20 de enero de $1790,{ }^{63}$ en la que se reconoce la propiedad intelectual ya no como un privilegio, sino como un derecho que debía ser "asegurado a su autor por la ley". No obstante, al establecerse un límite temporal a la propiedad sobre las ideas, lo que debería ser un derecho natural de propiedad en el sentido de Diderot, en la práctica se mantiene como un privilegio, ya no real, sino legal; la diferencia entre ambos es que mientras el primero perdura en el tiempo, el segundo, por ley, tendría su fecha de caducidad. El reconocimiento de la propiedad intelectual como un privilegio tolerado por la sociedad podría explicar por qué esta "clase" de propiedad, a diferencia de aquella sobre los recursos materiales, es limitada en el tiempo: es necesario favorecer el progreso de las luces mejorando las condiciones educativas y culturales. ${ }^{64}$

\section{La cláusula del progreso}

No obstante, Machlup y Penrose ${ }^{65}$ sugieren que la noción del contrato social les permitió a los franceses legitimar la patente como el resultado de un proceso de negociación (quid pro quo) y no como

63 "Le progrès des lumières, et par conséquent l'utilité publique se réunissent aux idées de justice distributive, pour exiger que la propriété d'un ouvrage soit assurée à l'auteur par la loi. En conséquence, toute personne convaincue d'avoir imprimé un livre pendant la vie d'un auteur, ou moins de dix ans après sa mort, sans son consentement exprès et par écrit, ou celui de ses ayans-cause, sera déclaré contrefacteur...". Véase, Bouchez, B. J. y Roux, P., Histoire parlementaire de la Révolution française ou Journal des Assambleées Nationales, depuis 1789 jusqu'en 1813, París, Paulin, 1834, p. 4. Esta propuesta fue también atribuida a Condorcet durante la época. Véase Lanthenas, François, De la liberté indéfinie de la presse, et de l'importance de ne soumettre la communication des pensées qu'a l'opinion publique: adressé et recommandé a toutes les sociétés patriotiques, populaires et fraternelles, de l'empire François, París, Visse \& Desenne, 1791, p. 6.

64 La propuesta Condorcet/Sieyès no fue aprobada en 1790, pero tres años más tarde se aprobó una versión similar a la misma. El objetivo de esta nueva versión no era para asegurar la responsabilidad de los autores, ni para proteger los intereses privados en el mercado del libro, sino como un mecanismo que, más allá de alentar y compensar la actividad intelectual, promoviera y asegurara la ilustración pública; véase Hesse, op. cit., p. 128.

65 Machlup, Fritz y Penrose, Edith, "The Patent Controversy in the Nineteenth Century", The Journal of Economic History, vol. 10, núm 1, 1950. 
SOBRE LOS FUNDAMENTOS DE LA PROPIEDAD INTELECTUAL

un privilegio. En este sentido, la propiedad intelectual también se ha justificado sobre argumentos que convergen alrededor de la llamada "cláusula del progreso", incluida por primera vez en la Constitución de los Estados Unidos de 1787, que le impuso al Congreso la obligación de "sancionar leyes dirigidas a fomentar el progreso de la ciencia y las artes útiles, asegurando a los autores e inventores, por un tiempo limitado, el derecho exclusivo sobre sus respectivos escritos y descubrimientos". ${ }^{66}$ La cláusula aparece también expresada en el orden internacional en el artículo 7o. del Acuerdo sobre los Aspectos de Propiedad Intelectual Relacionados con el Comercio (ADPIC) en los siguientes términos:

...la protección y la observancia de los derechos de propiedad intelectual deben contribuir a la promoción de la innovación tecnológica y a la transferencia y difusión de la tecnología, en beneficio recíproco de los productores y de los usuarios de conocimientos tecnológicos y de modo que favorezcan el bienestar social y económico y el equilibrio de derechos y obligaciones.

La cláusula del progreso se enfrenta al paradójico problema que encierra la idea de que para promover la creación es necesario res-

${ }^{66}$ La cláusula del progreso es considerada como la primera ley sustantiva en la historia legislativa de los Estados Unidos que reconoce derechos de propiedad a los autores e inventores sobre sus creaciones. Véase Ramsey, op. cit., p. 15. Esta cláusula se incorporó, posteriormente, en la Ley de Patentes de 1790 de los Estados Unidos. Algunos autores, como Campbell, derivan la historia del sistema de patentes de Estados Unidos desde el Statute of Monopolies inglés. Véase Campbell, Levin, The Patent System of the United States so far as it Relates to the Granting of Patents, Washington, D. C., Press of McGill and Wallace, 1891. Otros, como Ramsey, encuentran que existen elementos comunes (e. g., novedad como requisito de patentabilidad) entre la cláusula constitucional de 1787, la Ley de Patente de 1790 y el Statute of Monopolies. Véase Ramsey, op. cit., por su parte, Bracha califica a la ley de 1790 como un híbrido entre el sistema moderno de patentes y el sistema de privilegios inglés, que devino en un sistema de privilegio universalizado. Véase Bracha, op. cit. En este sentido, la Corte Suprema de los Estados Unidos ha utilizado el oxímoron "copyright privilege" en algunas decisiones. Véase Sony Corp. v. Universal City Studios, 464 U.S. 417 (1984); Watson v. Buck, 313 US 387 (1941), aunque en otros momentos ha reiteradamente utilizado el término "privilegio" para referirse a los derechos de propiedad intelectual. Veáse Grant $v$. Raymond, 31 US (6 Pet.) 218 (1832) y Eldred v. Ashcroft, 537 US 186 (2003). 
tringir el acceso a recursos intelectuales. ${ }^{67}$ Debemos entender que la creación intelectual no responde a un proceso individual y estático (romance), sino a un proceso dinámico, a un continuum en el que unas ideas llevan a otras ideas (realidad), en donde algunas son cruciales para el desarrollo de otras. Por tanto, la aplicación de la propiedad intelectual en su forma monopólica tradicional sería, cuando menos, caprichosa, pues al asignar al último eslabón de la cadena todo el valor de la creación, se desconocería el valor que sus predecesores tendrían por su trabajo.$^{68}$ En este sentido, existen teorías como la de Merges y Nelson ${ }^{69}$ y la de Lemley, ${ }^{70}$ para quienes existen situaciones en donde sería más importante favorecer un ambiente que tienda al desarrollo de mejoras que a la protección individual de "pioneros", pues estos últimos podrían estar incentivados a no llegar a acuerdos de licenciamiento con los creadores subsecuentes, obstruyendo la posible innovación. La patente sobre la máquina de vapor de Boulton y Watt, la patente de motor de automóviles de Selden, la patente del aeroplano de los hermanos Wright y el traslado de la industria química de Inglaterra a Suiza (donde la política contraria a las patentes permitió su desarrollo) son algunos de los ejemplos que permiten cuestionar la cláusula del progreso, pues en esos casos, en lugar de promoverse la innovación y la creación, se han inhibido o, al menos, ralentizado. Este problema no es sólo retórico, ${ }^{71}$ sino que empíricamente puede ser comprobado, como en el caso de la industria farmacéutica italiana, en la que la introducción del sistema de patentes en 1978 significó un retroceso en la producción de nuevos compuestos químicos con

67 Hettinger, op. cit.

68 Ibidem, p. 38.

69 Merges, Robert y Nelson, Richard, "On the Complex Economics of Patent Scope”, Columbia Law Review, vol. 90, núm. 4, 1990.

70 Lemley, Mark, "The Economics of Improvement in Intellectual Property Law", Texas Law Review, vol. 75, 1997.

71 Palmer considera que el supuesto incentivo que produce la patente no está claro, porque no se basa en estudios empíricos, sino que se asume como "moneda de ley". Véase Palmer, Tom, "Are Patents and Copyrights Morally Justified? The Philosophy of Property Rights and Ideal Objects", Harvard Journal of Law and Public Policy, vol. 13, núm. 3, 1990, p. 863. 
respecto al periodo anterior. ${ }^{72}$ Suiza, a pesar de las presiones internacionales, se negó a sancionar una ley de patentes en 1863, y no adoptó una sino hasta 1907. Francia, Alemania, Italia, Japón y Suecia también se resistieron patentar productos farmacéuticos hasta que sus respectivas industrias alcanzaron, a partir de 1960, cierto grado de desarrollo. ${ }^{73}$ Chang ha puesto de manifiesto cómo la política contraria a las patentes contribuyó al desarrollo de países como Suiza, permitiendo especialmente la incorporación de las ideas alemanas en las industrias química y farmacéutica y estimulando las inversiones directas extranjeras en el sector alimentario. ${ }^{74}$ En la práctica, los derechos de patente, por ejemplo, pueden representar, más que un incentivo, un obstáculo mediante, primero, el condicionamiento de las líneas de investigación al financiamiento de líneas donde la patentabilidad de las invenciones sea más probable; segundo, la aparición de anticommons debido a la excesiva fragmentación del conocimiento a través de las patentes; tercero, el otorgamiento de patentes excesivamente amplias, las cuales reivindican usos que van más allá de los descritos en los documentos de patente; cuarto, la excesiva judicialización de los derechos de patente, y quinto, el secretismo como práctica incentivada por la exigencia de la novedad como requisito para patentar. ${ }^{75}$

\section{La paradoja de Arrow}

Desde la perspectiva economicista se ha entendido que una vez que el conocimiento ha sido producido, su coste marginal de uso

72 Para una visión más completa del problema a nivel histórico y económico se sugiere ver el excelente recorrido realizado por Boldrin Michael y Levine David, Against Intellectual Monopoly, Cambridge, Cambridge University Press, 2008, p. 298.

73 Pretorius, Willem, "TRIPS and Developing Countries: How Level is the Playing Field?", en Drahos, Peter y Mayne, Ruth (eds.), Global Intellectual Property Rights: Knowledge, Access and Development, New York, Palgrave MacMillan Oxfam, 2002, 184.

${ }^{74}$ Chang Ha-Joon, Kicking away the Ladder: Development Strategy in Historical Perspective, London, Anthem Press, 2002, p. 18.

75 Wong, Sulan, "Patents and Scientific Research: Five Paradoxical Scenarios”, en Terán, Oswaldo y Aguilar, José (eds.), Societal Benefits of Freely Accessible Technologies and Knowledge Resource, Hershey, IGI Global, 2015. 


\section{SULAN WONG}

y de distribución es cero. Por lo tanto, sería ineficiente excluir a alguien de los beneficios que de él se pueden obtener, ${ }^{76}$ alcanzándose el óptimo con la distribución libre de información y conocimiento. ${ }^{77}$ Arrow, en un artículo publicado en 1962, coincide con Nelson en este aspecto ${ }^{78}$ pero sostiene que la utilización óptima del conocimiento no provee ningún incentivo para la inversión. ${ }^{79}$ Por ello, la restricción de acceso - aunque subóptima socialmente- es necesaria, pues crea una escasez artificial del recurso que permite extraer el valor económico del conocimiento y de la información, incentivando su producción. El problema está en que tanto los costes como los beneficios resultan exagerados, y no es fácil encontrar la medida o el balance apropiado. Como afirma Stiglitz, el poder monopólico genera rentas monopólicas, aunque el coste social de las distorsiones e ineficiencias de la propiedad intelectual puede sobrepasar los beneficios. La limitación del uso de la creación intelectual per se es uno de estos problemas, sobre el que quizá haya que prestar mucha atención, porque cuando se impide la diseminación y el uso del conocimiento, se ralentiza la continuidad de nuevas investigaciones, innovaciones sobre otras innovaciones, y ello puede obstruir el progreso científico-tecnológi-

76 Stiglitz, Joseph, "Knowledge as a Global Public Good", en Kaul, I. et al. (eds.), Global Public Goods, Nueva York, Oxford University Press, 1999.

77 Nelson, Richard, "The Simple Economics of Basic Scientific Research", The Journal of Political Economy, vol. 67, núm. 3, 1959, p. 306.

78 Arrow, Kenneth, "Economic welfare and the allocation of resources for invention", en The Rate and Direction of Inventive Activity: Economic and Social Factors, Princeton, Princeton University Press, 1962, pp. 616 y 617.

79 El argumento del incentivo en la patente se fortalece aún más cuando la industria científica afirma que no podría llevar a cabo investigación alguna si ésta no asegura la recuperación de la inversión más los beneficios derivados de la comercialización de la invención, algo que sólo puede ser realizado con el monopolio que confiere un resultado patentable. Véase Hettinger, op. cit., p. 49; Bonnet, Sébastien et al., "A Mitochondria-K+ Channel Axis Is Suppressed in Cancer and Its Normalization Promotes Apoptosis and Inhibits Cancer Growth", Cancer Cell, vol. 11, núm. 1, 2007; Canadians for Health Research, Dr. Evangelos Michelakis, Researcher of the month: Nov. 2010, disponible en: http://www.chrcrm.org/en/rotm/dr-evangelosmichelakis. 
co. $^{80}$ En resumen, se privilegia el beneficio subóptimo individual sobre el beneficio óptimo social. ${ }^{81}$

\section{CONCLUSIONES}

Responder a la pregunta sobre cuáles son los fundamentos que legitiman la propiedad intelectual es una tarea laboriosa, que para algunos permanece aún sin contestar, dadas las complejidades que encierran las teorías desde las que se intenta explicar sus fundamentos. Así, el iusnaturalismo no podría explicar la conformación del commons intelectual ex ante, en los mismos términos que Locke definió el commons material - esto es, inclusivo y positivo- porque los recursos intelectuales ya han sido apropiados previamente a la conformación del commons intelectual ex post. Por tanto, debería darse un proceso inverso de conformación de este commons, de privado a comunal, que dependería de la voluntad de los comuneros, para hacer disponible el commons intelectual ex ante. Además, si el trabajo le agrega valor al recurso comunal, como lo plantea Locke en su obra con respecto a la tierra inculta, y lo transforma en propiedad individual, los recursos intelectuales al responder al llamado "efecto red" adquieren más valor a medida que más personas lo usan.

Por otra parte, los planteamientos del utilitarismo tampoco resultan convincentes y lucen contradictorios, dado que al privilegiar el beneficio subóptimo individual sobre el beneficio óptimo social, la creación intelectual como un hecho fundado en la sociedad misma es a la vez negada - mediante el reconocimiento de derechos de propiedad - y reconocida - mediante el límite temporal a estos derechos- . Nos preguntamos si la búsqueda del equilibrio soportada en la utilidad pública en la práctica se termina diluyendo en el fundamento lockeano generalmente asociado a la propiedad intelec-

${ }^{80}$ Stiglitz, Joseph, Declaration of Joseph E. Stiglitz in Association for Molecular Pathology, et al. (plaintiff) v. United States Patent and Trademark Office et al. (defendants) 09 Civ. 4515, 2010.

81 Ello porque los costes sociales asociados a la toma de decisiones que pueden ser mayores que los beneficios alcanzados no son considerados. Véase Coase, R. H., "The Problem of Social Cost", Journal of Law and Economics, vol. 3, 1960. 
tual, utilizándose una fachada utilitarista para enmascarar pilares de base iusnaturalista.

Si la visión utilitarista de los derechos de propiedad intelectual no tiene asidero, quedaría únicamente la retórica del derecho del creador a su obra, algo que, como hemos visto, no es más sino una errada derivación de la teoría lockeana a la creación intelectual. Por tanto, deberíamos abandonar concepciones eufemísticas y considerar a los derechos de propiedad intelectual como lo que realmente son, como fueron concebidos en su origen, como privilegios otorgados por el Estado para garantizar la comercialización y explotación industrial de la creación intelectual.

AgRAdECIMIENTos. El presente trabajo fue realizado en el marco del proyecto de investigación Fondecyt de Iniciación 11150162 “Patentes e investigación científica: un estudio empírico sobre el ejercicio del derecho de libertad de investigación científica en las universidades chilenas que desarrollan I+D". Le damos las gracias a las personas que con sus comentarios y sugerencias ayudaron a mejorar este trabajo.

\section{BIBLIOGRAFÍA}

ArRow, Kenneth Joseph, "Economic Welfare and the Allocation of Resources for Invention", en The Rate and Direction of Inventive Activity: Economic and Social Factors, Princeton, Princeton University Press, 1962.

Ascarelli, Tulio, Teoría de la concurrencia y de los bienes inmateriales, trad. de S. Verder (caps. I-VI), y L. Suárez-Llanos (caps. VII y ss.), 3a. ed., Barcelona, Bosch, 1970.

Воввіо, Norberto, Locke e il diritto naturale, Torino, G. Gappichelli, 1963.

Boldrin, Michael y Levine, David K., Against Intellectual Monopoly, Cambridge, Cambridge University Press, 2008.

Bonnet, Sebastian et al., "A Mitochondria-K+ Channel Axis Is Suppressed in Cancer and Its Normalization Promotes Apoptosis and Inhibits Cancer Growth", Cancer Cell, vol. 11, núm. 1, 2007. 
Bouchez, B. J. y Roux, P., Histoire parlementaire de la Révolution française ou Journal des Assambleées Nationales, depuis 1789 jusqu'en 1813, París, Paulin, 1834, vol. 4.

Boyle, James, The Public Domain. Enclosing the Commons of the Mind, New Haven, Yale University Press, 2008.

BrachA, Oren, "The Commodification of Patents 1600-1836: How Patents Became Rights and why we Should Care", Loyola of Los Angeles Law Review, vol. 38, 2004-2005.

Campbell, Levin Hicks, The Patent System of the United States so far as it Relates to the granting of patents, Washington, D. C., Press of McGill and Wallace, 1891.

Canadians for Health Research, Dr. Evangelos Michelakis, Researcher of the month: Nov. 2010. Disponible en: http://www.chrcrm.org/en/rotm/dr-evangelos-michelakis.

CAPELla, Juan Ramón, Fruta prohibida: una aproximación históricoteorética al estudio del derecho y del Estado, 5a. ed., Madrid, Trotta, 1997.

ChANG, Ha-Joon, Kicking away the Ladder: Development Strategy in Historical Perspective, London, Anthem Press, 2002.

CoAse, Ronald Harry, "The Problem of Social Cost", Journal of Law and Economics, vol. 3, 1960.

Condorcet, M. J. A. N.-de-C., Marquis de, "Fragments sur la liberté de la presse", en Oeuvres de Condorcet, París, Didot, 1776, t. II, disponible en: http://archiviomarini.sp.unipi.it/78/.

CwIK, Bryan, "Labor as the Basis for Intellectual Property Rights", Ethic Theory Moral Practice, vol. 17, 2014.

Diderot, Dennis, Lettre sur le comerce de la librairie: Lettre historique et politique adressée à un magistrat sur le commerce de la librairie, son état ancien et actuel, ses règlemnets, ses privilèges, les permissions tacites, les censeurs, les colporteurs, le passage des ponts et autres objets relatifs à la police littéraire, ed. por C. Paillard, Chicoutimi: Université du Québec à Chicoutimi, 2002/1763, disponible en: http://classiques. uqac.ca/classiques/Diderot_denis/lettre_commerce_livre/let tre_com_livre.html. 
Drahos, Peter, A Philosophy of Intellectual Property, Aldershot, Dartmouth, 1996.

Federico, Pasquale Joseph, "Origin and Early History of Patents", Journal of the Patent Office Society, vol. 11, 1929.

GETz, L., "History of the Patentee's Obligations in Great Britain: Part I", Journal of the Patent Office Society, vol. 46, 1964.

HEsSE, Carla, "Enlightenment Epistemology and the Laws of Authorship in Revolutionary France, 1777-1793", Representations, núm. 30, 1990.

Hettinger, Edwin C., "Justifying Intellectual Property", Philosophy and Public Affairs, vol. 18, núm. 1, 1989.

Himanen, Pekka, The Hacker Ethic and the Spirit of the Information Age, New York, Random House, 2001.

Himma, Kenneth, "Toward a Lockean Moral Justification of Legal Protection of Intellectual Property", San Diego Law Review, vol. 49, núm. 1, 2013.

Hughes, Justin, "The Philosophy of Intellectual Property", Georgetown Law Journal, vol. 77, 1988.

Hulme, E. Wyndham, "History of the Patent System under the Prerogative and at Common Law", Law Quarterly Review, vol. 12, 1896.

Lanthenas, François, De la liberté indéfinie de la presse, et de l'importance de ne soumettre la communication des pensées qu'a l'opinion publique : adressé et recommandé a toutes les sociétés patriotiques, popu- laires et fraternelles, de l'empire François, París, Visse \& Desenne, 1791.

LEMLEY, Mark A., "The Economics of Improvement in Intellectual Property Law", Texas Law Review, vol. 75, 1997.

LEMLEY, Mark A., "Faith-Based Intellectual Property”, UCLA Law Review, 2015, vol. 62 .

LESSIG, Lawrence, "Introducción", en STALlman, Richard, Software libre para una sociedad libre, Madrid, Traficantes de Sueños, 2004. 
Locke, John, Segundo tratado sobre el gobierno civil, 1a. ed., trad. de Carlos Mellizo, Madrid, Alianza Editorial, 1990.

Machlup, Fritz y Penrose, Edith, "The Patent Controversy in the Nineteenth Century", The Journal of Economic History, vol. 10, núm 1, 1950.

MACPHERSON, C. B., The political Theory of possessive Individualism: Hobbes to Locke, New York, Oxford University Press, 1962.

Merges, Robert P., Justifying Intellectual Property, Cambridge, Harvard University Press, 2011.

Merges, Robert P. y Nelson, Richard R., "On the Complex Economics of Patent Scope”, Columbia Law Review, vol. 90, núm. 4, 1990.

MgbeojI, Ikechi, "The Juridical Origins of the International Patent System: Towards a Historiography of the Role of Patents in Industrialization", Journal of the History of International Law, vol. 5, 2003.

Moore, Adam, Intellectual Property and Information Control, New Brunswick, NJ Transaction Publishers, 2004.

Mossoff, Adam, "Saving Locke from Marx: The Labor Theory of Value in Intellectual Property Theory", Social Philosophy and Policy, vol. 29, núm. 2, 2012.

Nelson, Richard, "The Simple Economics of Basic Scientific Research", The Journal of Political Economy, vol. 67, núm. 3, 1959.

NetCRAFT, February 2018 Web Server Survey, 2018, disponible en: https://news.netcraft.com/archives/2018/02/13/february2018-web-server-survey.html

OGBuRn, William y Thomas, Dorothy, "Are Inventions Inevitable? A Note on Social Evolution", The Academy of Political Science, vol. 37, núm. 1, 1922.

Palmer, Tom G., "Are Patents and Copyrights Morally Justified? The Philosophy of Property Rights and Ideal Objects", Harvard Journal of Law and Public Policy, vol. 13, núm. 3, 1990. 
Pollack, Malla, "The Multiple Unconstitutionality of Business Method Patents: Common Sense, Congressional Choice, and Constitutional History", Rutgers Computer \& Technology Law Journal, vol. 28, 2002.

Prager, Frank D., "Brunelleschi's Patent", Journal of the Patent Office Society, vol. 28, 1946.

Pretorius, Willem, "TRIPS and Developing Countries: How Level is the Playing Field?", en Drahos, Peter y MaYne, Ruth (eds.), Global Intellectual Property Rights: Knowledge, Access and Development, New York, Palgrave MacMillan Oxfam, 2002.

RAMSEY, George, "The Historical Background of Patents", Journal of the Patent Office Society, vol. 18, 1936.

Rose, Carol, Property \& Persuasion: Essays on the History, Theory, and Rhetoric of Ownership, Boulder, Westview Press, 1994.

Spooner, Lysander, The Law of Intellectual Property; or an essay on the right of authors and inventors to a perpetual property in their ideas, Boston, Bela Marsh, 1885, vol. I.

Stiglitz, Joseph, "Knowledge as a Global Public Good", en I. Kaul et al. (eds.), Global Public Goods, New York, Oxford University Press, 1999.

Stiglitz, Joseph, Declaration of Joseph E. Stiglitz in Association for Molecular Pathology, et al. (plaintiff) v. United States Patent and Trademark Office et al. (defendants) 09 Civ. 4515, 2010.

Tully, James, An approach to Political Philosophy. Locke in Contexts, New York, Cambridge University Press, 1993.

Willinsky, John, The Intellectual Properties of Learning: A Prehistory from Saint Jerome to John Locke, University of Chicago Press, 2017.

Wong, Sulan, "Patents and Scientific Research: Five Paradoxical Scenarios", en TERÁn, Oswaldo y AGuILAR, José (eds.), Societal Benefits of Freely Accessible Technologies and Knowledge Resource, Hershey, IGI Global, 2015. 